\title{
Serum neutrophil gelatinase-associated lipocalin for predicting anemia of inflammation in children with urinary tract infection
}

\author{
GRAZYNA JADWIGA KRZEMIEN', MALGORZATA PANCCZYK-TOMASZEWSKA ${ }^{l}$, \\ AGNIESZKA TURCZYN ${ }^{1}$, URSZULA DEMKOW ${ }^{2}$ IWONA KOTUEA ${ }^{2}$, AGNIESZKA SZMIGIELSKA ${ }^{l}$ \\ ${ }^{1}$ Department of Pediatrics and Nephrology, Medical University of Warsaw, Warsaw, Poland \\ ${ }^{2}$ Department of Laboratory Diagnostics and Clinical Immunology of Developmental Age, Medical University of Warsaw, Warsaw, Poland
}

\begin{abstract}
Anemia of inflammation (IA), the second most common cause of childhood anemia, results from macrophage iron sequestration and impaired erythropoiesis. Neutrophil gelatinase-associated lipocalin $(N G A L)$ plays an important role in innate microbial immunity through its influence on intracellular iron homeostasis and inhibition of erythropoiesis. The predictive value of NGAL in IA was assessed in 74 children (age $6.30 \pm 3.64$ months) with the first episode of urinary tract infection (UTI). Anemia of inflammation was found in $50 \%$ of children, including those with non-febrile UTI, and delayed onset of anemia was observed in $20 \%$ of children. There were no differences in NGAL levels between the anemic and non-anemic children, and no correlations between NGAL and hemoglobin (HGB) levels and red blood cell $(R B C)$ count. In multivariate logistic regression analysis elevated $C$-reactive protein $(C R P)$ was only independently associated with the presence of anemia in children with UTI [OR (95\% $C I): 1.128$ (1.005-1.265), $p=0.040]$. In stepwise multiple analysis age independently correlated with $R B C(\beta=0.051, p=0.001)$, while CRP independently correlated with $H G B(\beta=-0.037, p=0.027)$ and $R B C(\beta=-0.022, p=0.014)$. ROC analysis demonstrated better diagnostic profiles for CRP, procalcitonin (PCT) and fever duration for predicting the risk of IA than NGAL (AUC: 0.690, 0.669, 0.678 vs. 0.638 , respectively). Despite the increase in HGB levels after 4-5 weeks from the onset of UTI, HGB values were still significantly lower in the anemic than in non-anemic children. NGAL was not useful for predicting IA in UTI, since its diagnostic value was inferior to conventional inflammatory markers.
\end{abstract}

Key words: NGAL, anemia, anemia of inflammation, urinary tract infection, children.

(Cent Eur J Immunol 2021; 46 (4): 456-462)

\section{Introduction}

Anemia of inflammation (IA), the second most common cause of childhood anemia, results from macrophage iron sequestration and impaired erythropoiesis [1]. In children IA is commonly observed in the course of systemic bacterial, fungal, viral, and parasitic infections, less frequently in chronic inflammatory diseases and neoplasms [2]. The incidence of IA associated with acute infectious diseases is estimated at $29.9-55.7 \%$ in children and $40.6 \%$ in adults [3-5]. It is often mistaken for iron deficiency anemia (IDA), the most common cause of anemia in children, which is usually observed around 6 months of age [4, 6]. Iron homeostasis is controlled by various mechanisms at systemic and cellular levels. Systemic iron homeostasis is mainly regulated by hepcidin, a peptide hormone synthe- sized in hepatocytes. In acute bacterial infections, upregulation of hepcidin inhibits the intestinal absorption of iron and its release from macrophage iron stores to restrict bacterial access to iron as a part of the host's natural defense against pathogens $[1,7]$.

Neutrophil gelatinase-associated lipocalin (NGAL) mediates transferrin- and hepcidin-independent iron delivery pathways. NGAL belongs to the lipocalin superfamily of more than 20 small molecule proteins transporting various ligands [8]. Originally identified in activated neutrophils, it is an acute phase protein that plays a key role in innate microbial immunity, influences intracellular iron homeostasis, and inhibits erythropoiesis [3]. The most important ligands for NGAL are siderophores, small iron-containing hydrophobic molecules. Many bacteria and fungi secrete

Correspondence: Agnieszka Szmigielska, MD, PhD, Department of Pediatrics and Nephrology, Medical University of Warsaw, Warsaw, Poland, e-mail: agnieszka.szmigielska@wum.edu.pl

Submitted: 15.11.2021; Accepted: 13.12.2021 
siderophores to "steal" iron from the host's iron transporting proteins, i.e. transferrin and lactoferrin. NGAL binds bacterial siderophores to prevent bacteria from obtaining iron and as a result exerts bacteriostatic effect [9]. Moreover, NGAL regulates iron transport to and from the cell. After binding to the membrane receptor, NGAL is internalized by the cell either as a single protein (Apo-NGAL) that removes the excess iron from the cell or as a complex with iron-binding siderophores (Holo-NGAL) that supplies iron to the cell [10]. In the bone marrow, NGAL inhibits erythropoiesis by inducing apoptosis and influencing maturation and differentiation of erythroid progenitor cells $[11,12]$. The pathogenesis of IA in the course of systemic infections results from restricted iron availability for erythropoiesis and direct suppressive effects of NGAL and other pro-inflammatory cytokines on erythropoiesis, as well as via alterations of the erythrocyte membrane by pro-inflammatory cytokines that impair its survival [1].

In healthy people, NGAL is synthesized in small amounts in various organs. It is released from tissues and activated neutrophils and macrophages in response to cellular stress, such as ischemia, hypoxia, acute kidney injury (AKI), infection, inflammation or neoplastic transformation [9, 13]. NGAL undergoes glomerular filtration with subsequent reabsorption in the proximal tubule. Proximal tubule damage causes an increase in the concentration of NGAL in the urine, while damage to the distal part of the nephron causes increased synthesis and release of NGAL into the blood and the urine [13]. NGAL is a well-known diagnostic and prognostic marker of AKI [13-15] and chronic kidney disease (CKD) [16]. The clinical usefulness of NGAL has been demonstrated in systemic infections [9], including urinary tract infections (UTI) in children [17-19]. So far, few studies investigating the relationship between NGAL and the occurrence of anemia in systemic infections have been published [5], including only a single report in children [3]. Since UTI is one of the most com- mon bacterial infections in children [20], investigation of the relationship between NGAL and anemia can contribute to the risk assessment of IA and prediction of the severity of IA in children with UTI.

The aim of this study was to assess the predictive value of NGAL in IA in children with the first episode of UTI.

\section{Material and methods}

\section{Study population}

In this single center, cross-sectional study we assessed clinical data of 74 children with the first episode of UTI, treated in a tertiary center of pediatric nephrology. The inclusion criteria were: age from 2 months to 15 months and the first episode of febrile or non-febrile UTI. The exclusion criteria were: age, negative urinalysis or urine culture in children admitted to the hospital with the suspicion of UTI, concomitant other acute infectious disease, history of previous UTI or iron preparation intake within the last 3 months, and CKD [21]. Based on hemoglobin (HGB) level the children were divided into anemia $(n=37)$ and non-anemia $(n=37)$ groups. The study design is displayed in the flow diagram in Figure 1.

This study was approved by the local Bioethics Committee for Human Research (KB168/11). All parents gave written informed consent. All procedures performed in the study were in accordance with the guidelines of the 2013 Declaration of Helsinki.

\section{Clinical and laboratory data}

The following clinical and laboratory data were evaluated on admission: age (months), sex, the presence and duration of fever (days) before the admission to the hospital, the results of urinalysis and urine culture, complete blood count (CBC), serum NGAL, C-reactive protein (CRP), procalcitonin $(\mathrm{PCT})$, and creatinine $(\mathrm{Cr})$ levels. Urine

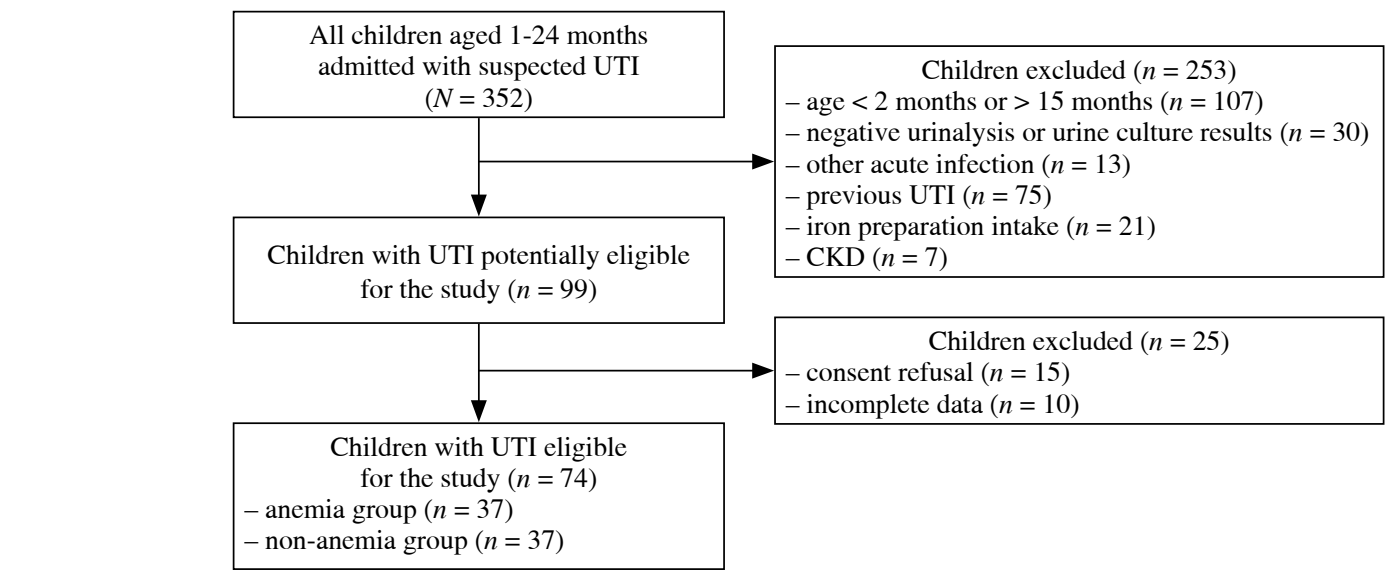

UTI - urinary tract infection, CKD - chronic kidney disease

Fig. 1. Study flow diagram 
Table 1. Reference range of red blood indices for children aged 2 months to 15 months

\begin{tabular}{lcccc}
\hline $\begin{array}{l}\text { Age } \\
(\mathbf{m o n t h s})\end{array}$ & $\begin{array}{c}\text { RBC } \\
(\mathbf{\times} \mathbf{1 0} / \boldsymbol{\mu l})\end{array}$ & $\begin{array}{c}\text { HGB } \\
(\mathbf{g} / \mathbf{d l})\end{array}$ & $\begin{array}{c}\text { MCV } \\
(\mathbf{f l})\end{array}$ & $\begin{array}{c}\text { MCH } \\
(\mathbf{p g})\end{array}$ \\
\hline 2 & $3.7-5.0$ & $10.0-13.5$ & $80-96$ & $27-31$ \\
\hline $3-5$ & $3.2-4.3$ & $9.5-13.0$ & $73-86$ & $27-34$ \\
\hline 6 & $3.8-5.0$ & $10.0-13.0$ & $73-86$ & $27-34$ \\
\hline $7-8$ & $3.8-5.0$ & $10.0-13.0$ & $73-86$ & $27-34$ \\
\hline $9-11$ & $4.0-5.3$ & $10.5-13.0$ & $73-86$ & $27-34$ \\
\hline $12-15$ & $4.2-5.5$ & $11.0-14.0$ & $72-88$ & $24-30$ \\
\hline
\end{tabular}

$R B C$ - red blood cells, HGB - hemoglobin, MCV - mean corpuscular volume, $\mathrm{MCH}-$ mean corpuscular hemoglobin

specimens were obtained by bladder catheterization. UTI diagnosis was made based on the presence of both pyuria and positive urine culture. Pyuria was defined as $\geq 5$ white blood cells (WBC)/high powered field on a centrifuged specimen of urine, and significant bacteriuria as pure bacterial growth $\geq 50,000$ colony-forming units $(\mathrm{CFU}) / \mathrm{ml}$ or $\geq 10,000 \mathrm{CFU} / \mathrm{ml}$, if concomitant fever and pyuria were present [22]. Fever was defined as body temperature of at least $38.0^{\circ} \mathrm{C}$ within 24 hours of presentation.

Anemia was defined as a reduction in HGB values below the normal range for the child's age. Anemia of inflammation was defined as anemia with normal mean corpuscular volume (MCV) and mean corpuscular hemoglobin $(\mathrm{MCH})$ values during infection and increase in red blood cell (RBC) and HGB values after treatment of UTI without iron supplementation. The reference range of hematological indices evaluated in the local laboratory are presented in Table 1. The reference ranges of biochemical indices were as follows: CRP $\leq 1.0 \mathrm{mg} / \mathrm{dl}$, PCT $<0.05 \mathrm{ng} / \mathrm{ml}$, and $\mathrm{Cr} \leq 0.4 \mathrm{mg} / \mathrm{dl}$. CBC was repeated on the $3^{\text {rd }}$ and $10^{\text {th }}$ day of antibiotic treatment, and after 4-5 weeks from the onset of the disease.

Serum samples for measurement of NGAL levels were collected prior to the initiation of antibiotic treatment, immediately centrifuged and stored at $-80^{\circ} \mathrm{C}$ until further analysis. NGAL levels were measured by a commercially available enzyme-linked immunosorbent assay (ELISA) kit (Human Lipocalin-2/NGAL; Cat. No. RD 191102200R; Bio Vendor Laboratory Medicine, Inc. Brno, Czech Republic). The detection limit for NGAL was 0.3-10 ng/ml. CBC was examined by an XN-1000 SA-01 automated hematology analyzer (Sysmex), and biochemical variables were measured using VITROS 5600 Integrated System (Ortho Clinical Diagnostics), except PCT (Vidas PC Blue, BioMérieux).

\section{Imaging data}

Ultrasonography (US) of the kidney and bladder was performed after admission. Hydronephrosis (HN) was classified according to the Society for Fetal Urology classifi- cation [23]. Voiding cystography (VCUG) was performed in children with abnormal findings on US or atypical UTI 2-3 weeks after UTI treatment. The severity of vesicoureteral reflux (VUR) was classified according to the International Reflux Study Committee classification [24].

\section{Statistical analysis}

Statistical analysis was performed using Statistica 13.3 PL software (StatSoft, Tulsa, OK, USA). Continuous variables were presented as mean \pm standard deviation (SD) or median (interquartile range), and categorical variables were presented as absolute frequencies and percentages. The normality of the data was assessed by the Shapiro-Wilk test. Student's $t$-test, the Mann-Whitney $U$-test, and the $\chi^{2}$ test were used to analyze data between the two groups. Paired Student's $t$-test was used to compare the increments of HGB values between the measurements. Correlations between clinical and laboratory variables and RBC indices were evaluated by linear regression analysis or Spearman's rank correlation, respectively. Stepwise multiple linear regression analysis was used to identify the linear relationship between clinical and laboratory variables and RBC and HGB values. Receiver operating characteristic (ROC) analysis was performed. The area under the curves (AUC) was calculated to determine the best cut-off values of the analyzed variables. Odds ratio (OR) and 95\% confidence intervals (CI) were calculated by univariate logistic regression analysis to identify variables associated with the presence of anemia. To determine independent risk factors of anemia, the variables related to anemia in univariate logistic regression were entered into a multivariate logistic regression model. $P$ values less than 0.05 were considered statistically significant.

\section{Results}

We evaluated 74 children (40 girls, 34 boys; mean age $6.30 \pm 3.64$ months) with the first episode of UTI. Most of them were under the age of 12 months $(n=67,90.5 \%)$. Febrile UTI was diagnosed in $57(77 \%)$ children, non-febrile UTI in 17 (23\%) children. Escherichia coli was isolated in $68(91.9 \%)$ patients, Klebsiella pneumoniae in 3 patients, Klebsiella oxytoca, Pseudomonas aeruginosa, and Enterococcus faecalis in one each. Based on US and VCUG, congenital anomalies of the kidney and urinary tract (CAKUT) were diagnosed in $14(18.9 \%)$ children. VUR was detected in $7(11.5 \%)$ patients undergoing VCUG $(n=61,82.4 \%)$. VUR grade 2 was found in 4 patients, VUR grade $4-5$ in three. A duplicated collecting system was found in 5 patients, $\mathrm{HN}$ in 3 patients (severe $\mathrm{HN}$ in two, mild $\mathrm{HN}$ in one), megaureter and kidney dysplasia in one each.

Baseline clinical and laboratory data in the study groups are presented in Table 2. The study groups did not differ in terms of age, sex, presence of fever or CAKUT, 
Table 2. Baseline clinical and laboratory data on admission in the study groups (significant differences in bold)

\begin{tabular}{lccc}
\hline Variables & $\begin{array}{c}\text { Anemia } \\
(\boldsymbol{n}=\mathbf{3 7})\end{array}$ & $\begin{array}{c}\text { Non-anemia } \\
(\boldsymbol{n}=\mathbf{3 7})\end{array}$ & $\boldsymbol{P}$ value \\
\hline Age (months) & $6.18 \pm 4.20$ & $6.42 \pm 3.02$ & 0.776 \\
\hline Female $(\%)$ & $19(51.3)$ & $21(56.7)$ & 0.605 \\
\hline Fever presence $(\%)$ & $32(86.5)$ & $25(67.6)$ & 0.066 \\
\hline Fever duration $($ days $)$ & $3.03 \pm 1.83$ & $1.81 \pm 1.70$ & $\mathbf{0 . 0 0 4}$ \\
\hline CAKUT $(\%)$ & $7(18.9)$ & $7(18.9)$ & 1.00 \\
\hline Creatinine $(\mathrm{mg} / \mathrm{dl})$ & $0.27 \pm 0.07$ & $0.28 \pm 0.09$ & 0.473 \\
\hline NGAL $(\mathrm{ng} / \mathrm{ml})$ & $143.54 \pm 72.00$ & $113.80 \pm 75.28$ & 0.087 \\
\hline WBC $\left(\times 10^{3} / \mu \mathrm{l}\right)$ & $18.59 \pm 6.65$ & $18.12 \pm 5.90$ & 0.749 \\
\hline Neutrophils $(\%)$ & $48.58 \pm 16.68$ & $44.89 \pm 19.59$ & 0.386 \\
\hline CRP $(\mathrm{mg} / \mathrm{dl})$ & $7.00(2.80 ; 14.90)$ & $3.40(1.70 ; 6.60$ & $\mathbf{0 . 0 0 5}$ \\
\hline PCT $(\mathrm{ng} / \mathrm{ml})$ & $0.73(0.11 ; 2.05)$ & $0.13(0.06 ; 0.49)$ & $\mathbf{0 . 0 1 5}$ \\
\hline
\end{tabular}

CAKUT - congenital anomalies of the kidney and urinary tract, $N G A L$ - neutrophil gelatinase-associated lipocalin, WBC - white blood cells, $C R P-C$-reactive protein, $P C T$ - procalcitonin

or Cr and WBC levels. However, two children demonstrated a slight and short-term increase in $\mathrm{Cr}$ level $(0.5 \mathrm{mg} / \mathrm{dl}$ and $0.6 \mathrm{mg} / \mathrm{dl}$, respectively) associated with high fever and dehydration. The mean NGAL level was higher in the anemia group than in the non-anemia group, but the difference was not significant. Five (13.5\%) children with anemia had non-febrile UTI. The mean fever duration was significantly longer and the median CRP and PCT levels were significantly higher in the anemia group compared to the non-anemia group. In 22 (29.7\%) of children, anemia was diagnosed on admission, in $9(12.2 \%)$ children on the $3^{\text {rd }}$ day, and in $6(8.1 \%)$ children on the $10^{\text {th }}$ day of treatment.

The comparison of RBC indices on admission and after 4-5 weeks from the onset of UTI in the study groups are presented in Table 3 . The study groups did not differ in terms of $\mathrm{MCV}$ and $\mathrm{MCH}$. The mean levels of $\mathrm{RBC}$ and HGB on admission were significantly lower in the anemia group compared to the non-anemia group. After 4-5 weeks
Table 3. Comparison of red blood cell indices during infection and after discharge from the hospital in the study groups (significant differences in bold)

\begin{tabular}{lccc}
\hline Variables & $\begin{array}{c}\text { Anemia } \\
(\boldsymbol{n}=\mathbf{3 7})\end{array}$ & $\begin{array}{c}\text { Non-anemia } \\
(\boldsymbol{n}=\mathbf{3 7})\end{array}$ & $\boldsymbol{P}$ value \\
\hline \multicolumn{4}{c}{ On admission } \\
\hline $\mathrm{RBC}\left(\times 10^{6} / \mu \mathrm{l}\right)$ & $3.56 \pm 0.34$ & $4.08 \pm 0.36$ & $\mathbf{0 . 0 0 0}$ \\
\hline $\mathrm{HGB}(\mathrm{g} / \mathrm{dl})$ & $9.80 \pm 0.74$ & $11.05 \pm 0.57$ & $\mathbf{0 . 0 0 0}$ \\
\hline $\mathrm{MCV}(\mathrm{fl})$ & $84.76 \pm 5.31$ & $82.72 \pm 5.64$ & 0.113 \\
\hline $\mathrm{MCH}(\mathrm{pg})$ & $27.69 \pm 2.03$ & $27.19 \pm 1.96$ & 0.256 \\
\hline \multicolumn{5}{c}{ After $4-5$ weeks from the onset of UTI } \\
\hline RBC $\left(\times 10^{6} / \mu \mathrm{l}\right)$ & $4.08 \pm 0.29$ & $4.23 \pm 0.42$ & 0.158 \\
\hline $\mathrm{HGB}(\mathrm{g} / \mathrm{dl})$ & $10.92 \pm 0.56$ & $11.44 \pm 0.77$ & $\mathbf{0 . 0 1 7}$ \\
\hline $\mathrm{MCV}(\mathrm{fl})$ & $82.12 \pm 4.75$ & $82.96 \pm 5.21$ & 0.583 \\
\hline $\mathrm{MCH}(\mathrm{pg})$ & $26.81 \pm 1.59$ & $27.09 \pm 1.67$ & 0.572 \\
\hline
\end{tabular}

$R B C$ - red blood cells, $H G B$ - hemoglobin, $M C V$ - mean corpuscular volume, $M C H$ - mean corpuscular hemoglobin, UTI - urinary tract infection

from the onset of UTI, no difference in the mean level of RBC between two groups was observed. However, despite the increase in HGB levels in both groups, the mean levels of HGB were still significantly lower in the anemia group than in the non-anemia group.

Ten $(27 \%)$ children with anemia had the lowest values of HGB on admission, thirteen $(35 \%)$ on the $3^{\text {rd }}$ day, and fourteen $(38 \%)$ on the $10^{\text {th }}$ day of treatment. At discharge $\left(10^{\text {th }}\right.$ day), $23(62.2 \%)$ of those children demonstrated anemia, and after 4-5 weeks from the onset of UTI anemia was present only in $1(2.7 \%)$ child. The mean decrease of HGB level during infection was $0.53 \pm 0.61 \mathrm{~g} / \mathrm{dl}$ in the anemia group and $0.20 \pm 0.32 \mathrm{~g} / \mathrm{dl}$ in the non-anemia group $(p=0.005)$. WBC, CRP, and PCT levels were the highest on admission. They gradually decreased and were normal on the $10^{\text {th }}$ day of treatment (data not shown).

The correlations of clinical and laboratory data on admission with RBC indices are presented in Table 4.

Table 4. Correlations of clinical and laboratory data on admission with red blood cell indices (significant correlations in bold)

\begin{tabular}{|c|c|c|c|c|c|c|c|c|}
\hline \multirow[t]{2}{*}{ Variable } & \multicolumn{2}{|c|}{$\begin{array}{c}\text { RBC } \\
\left(\times 10^{6} / \mu \mathrm{l}\right)\end{array}$} & \multicolumn{2}{|c|}{$\begin{array}{l}\text { HGB } \\
\text { (g/dl) }\end{array}$} & \multicolumn{2}{|c|}{$\begin{array}{c}\text { MCV } \\
\text { (fl) }\end{array}$} & \multicolumn{2}{|c|}{$\begin{array}{c}\text { MCH } \\
\text { (pg) }\end{array}$} \\
\hline & $r$ & $p$ & $r$ & $p$ & $r$ & $p$ & $r$ & $p$ \\
\hline Fever (days) & -0.01 & 0.903 & -0.19 & 0.100 & -0.20 & 0.093 & -0.25 & 0.036 \\
\hline NGAL (ng/ml) & -0.01 & 0.959 & -0.13 & 0.272 & -0.10 & 0.406 & -0.16 & 0.163 \\
\hline $\mathrm{WBC}\left(\times 10^{3} / \mu \mathrm{l}\right)$ & 0.06 & 0.604 & -0.01 & 0.941 & -0.03 & 0.769 & -0.16 & 0.170 \\
\hline CRP (mg/dl) & -0.11 & 0.367 & -0.27 & 0.018 & -0.15 & 0.194 & -0.19 & 0.102 \\
\hline PCT (ng/ml) & -0.09 & 0.487 & -0.16 & 0.196 & -0.12 & 0.332 & -0.06 & 0.637 \\
\hline
\end{tabular}

$\overline{R B C}$ - red blood cells, $H G B$ - hemoglobin, $M C V$ - mean corpuscular volume, $M C H$ - mean corpuscular hemoglobin, NGAL - neutrophil gelatinase-associated lipocalin, WBC-white blood cells, CRP - C-reactive protein, $P C T$ - procalcitonin 
Table 5. Diagnostic accuracy of clinical and laboratory data for predicting anemia in children with urinary tract infection (significant determinants in bold)

\begin{tabular}{lcccccc}
\hline Variable & AUC $(\mathbf{9 5 \%}$ CI) & P value & Cut-off & Sensitivity $(\boldsymbol{\%})$ & Specificity (\%) & ACC (\%) \\
\hline Fever $($ days $)$ & $0.678(0.556-0.801)$ & $\mathbf{0 . 0 0 4}$ & 3.00 & 67.6 & 64.9 & 66.2 \\
\hline NGAL $(\mathrm{ng} / \mathrm{ml})$ & $0.638(0.510-0.767)$ & $\mathbf{0 . 0 3 4}$ & 123.60 & 64.9 & 64.9 & 64.9 \\
\hline WBC $\left(\times 10^{3} / \mu 1\right)$ & $0.505(0.360-0.630)$ & 0.941 & - & - & - & - \\
\hline CRP $(\mathrm{mg} / \mathrm{dl})$ & $0.690(0.568-0.812)$ & $\mathbf{0 . 0 0 2}$ & 9.00 & 45.9 & 91.9 & 68.9 \\
\hline PCT $(\mathrm{ng} / \mathrm{ml})$ & $0.669(0.540-0.798)$ & $\mathbf{0 . 0 1 0}$ & 0.41 & 67.6 & 72.2 & 70.0 \\
\hline
\end{tabular}

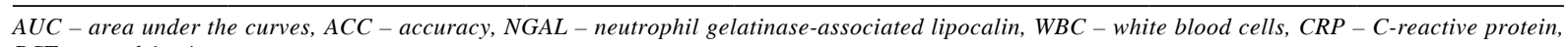
$P C T$ - procalcitonin

Table 6. Univariate and multivariate logistic regression analysis for predicting anemia in children with urinary tract infection (significant determinants in bold)

\begin{tabular}{lcccc}
\hline Variables & \multicolumn{2}{c}{ Univariate } & \multicolumn{2}{c}{ Multivariate } \\
\cline { 2 - 5 } & OR $(\mathbf{9 5 \%}$ CI) & $\boldsymbol{P}$ value & OR $(\mathbf{9 5 \%}$ CI) & $\boldsymbol{P}$ value \\
\hline Fever duration (days) & $1.470(1.115-1.938)$ & $\mathbf{0 . 0 0 6}$ & $1.372(0.987-1.908)$ & 0.060 \\
\hline NGAL $(\mathrm{ng} / \mathrm{ml})$ & $1.006(0.999-1.012)$ & 0.090 & $0.999(0.991-1.008)$ & 0.867 \\
\hline WBC $\left(\times 10^{3} / \mu \mathrm{l}\right)$ & $1.012(0.940-1.090)$ & 0.745 & - & - \\
\hline CRP $(\mathrm{mg} / \mathrm{dl})$ & $1.131(1.040-1.231)$ & $\mathbf{0 . 0 0 4}$ & $1.128(1.005-1.265)$ & $\mathbf{0 . 0 4 0}$ \\
\hline PCT $(\mathrm{ng} / \mathrm{ml})$ & $1.029(0.944-1.122)$ & 0.509 & - & - \\
\hline NGAL - neutrophil gelatinase-associated lipocalin, WBC - white blood cells, CRP - C-reactive protein, PCT - procalcitonin &
\end{tabular}

There were no correlations between NGAL and RBC indices. In stepwise multiple linear regression analysis age independently correlated with RBC count $(\beta=0.051$, $p=0.001)$, while CRP independently correlated with RBC count and HGB level $(\beta=-0.022, p=0.014 ; \beta=-0.037$, $p=0.027$, respectively) when fever duration, NGAL, WBC and PCT were included as independent variables. ROC analysis demonstrated that fever duration, CRP, and PCT had better diagnostic profiles for predicting the risk of anemia than NGAL (Table 5). The AUC and accuracy (ACC) were higher for PCT, CRP and fever duration than for NGAL. Univariate logistic regression analysis demonstrated that fever duration and CRP were associated with the presence of anemia in children with UTI. In multivariate analysis only elevated CRP was independently associated with the presence of anemia in children with febrile and non-febrile UTI (Table 6).

\section{Discussion}

In this study, IA was found in $50 \%$ of children with UTI. NGAL level was higher in the anemia group than in the non-anemia group, but the difference was not significant. No correlation between NGAL and RBC indices was observed. The diagnostic profile for predicting anemia for NGAL was inferior to those for CRP, PCT and fever duration.

In contrast to the present study, Lee et al. observed a significantly higher plasma NGAL level in children with febrile UTI and anemia compared to those without anemia, and negative correlations of NGAL and CRP with RBC count and HGB level [3]. Similar to our study, the diagnostic profile for predicting anemia for NGAL (AUC 0.641) was inferior to that for CRP (AUC 0.668). In a study by Choi et al., anemic patients had a significantly higher plasma NGAL level in comparison to non-anemic patients, and there was a negative correlation between NGAL and RBC count and HGB level [5]. The utility of NGAL for diagnosing anemia was higher (AUC 0.712) than high-sensitivity CRP (AUC 0.649), but lower than the erythrocyte sedimentation rate (ESR) (AUC 0.751). Also in this study, an increase in NGAL level was an independent risk factor for the development of IA in patients with systemic infections. The results of the above studies suggest an adverse effect of circulating NGAL on RBC maturation in the bone marrow. However, in both studies, the diagnostic profile of NGAL for predicting IA was comparable to or lower than that of conventional inflammatory markers.

Severe UTI complicated with APN may lead to acute tubulo-interstitial kidney injury and subclinical or symptomatic AKI [25, 26]. The increase in NGAL level in the blood and urine is observed 1 to 3 hours after kidney injury [13]. In our study, children with UTI did not show symptoms of AKI. Only in 2 patients was a slight and short-term increase in serum creatinine associated with high fever and dehydration found. NGAL increase in systemic infections may also be related to the occurrence of subclinical AKI due to a low serum iron (SI) level [2]. Iron deficiency re- 
duces the ability of HGB to transport oxygen to tissues and can lead to hypoxia and cell damage. Güneş et al. observed significantly higher levels of NGAL and other biomarkers of early kidney injury in children with IDA compared to healthy children, and a negative correlation of NGAL with RBC indices [27]. According to the authors, chronic hypoxia in IDA causes mild, subclinical kidney injury that can only be diagnosed by AKI markers in the urine. It can be assumed that a decrease in SI concentration and tissue hypoxia may be the cause of subclinical kidney injury also in children with a severe course of UTI. In the present study MCV and $\mathrm{MCH}$ values (surrogate markers of predicting iron deficiency) did not indicate significant iron deficiency in the studied children.

The course of UTI and the occurrence of IA may be influenced by the etiology of infection. Some bacteria and fungi, to overcome the bacteriostatic effect of NGAL, have developed various defense mechanisms. Pseudomonas aeruginosa and Klebsiella pneumoniae secrete "clandestine" siderophores that are not recognized by NGAL. Some strains of Klebsiella pneumoniae and Escherichia coli enhance the biological activity of siderophores through their glycosylation. Other bacteria have developed alternative methods of obtaining iron that are not based on siderophores [9]. In our study, Escherichia coli was the cause of UTI in $92 \%$ of children. We have only recorded isolated cases of UTIs caused by other bacteria. Therefore, in our material the etiology of infection did not influence the severity of IA.

The symptoms of anemia of inflammation in systemic infections may not show until several days after the onset of infection. The delayed onset of anemia may be the reason why IA is not diagnosed in some children with UTI. IA can vary in severity, from mild to severe, and may increase with infection. In our study, in $8.1 \%$ of children anemia was diagnosed on the $10^{\text {th }}$ day of infection. In $38 \%$ of anemic children, the lowest HGB values were found only on the $10^{\text {th }}$ day of UTI. After 4-5 weeks from the onset of the disease, the percentage of children with anemia was $2.7 \%$. Sipahi et al. found a significant reduction in HGB concentration in $55.7 \%$ of children with acute infectious diseases by $0.1-2.4 \mathrm{~g} / \mathrm{dl}$ (mean $0.6 \pm 0.5 \mathrm{~g} / \mathrm{dl}$ ) on the $3^{\text {rd }}$ day of infection compared to the value on the first day of the study. On study day $15,53 \%$ of patients still had low HGB levels. IA may persist for up to 3 months, usually resolves spontaneously after the infection is resolved and, unlike IDA, does not require treatment [4].

Our study has some limitations. This was a single center study with a small sample size. As HGB levels depend on the age of the child, anemia was defined as a reduction in HGB levels below the normal range for the child's age. In order to minimize this confounding factor, we excluded children below 2 months of age and over 15 months of age. We did not determine the levels of SI. The diagnosis of IA based solely on RBC indicators may be biased. In children with IA, MCV and $\mathrm{MCH}$ may be reduced due to the use of iron for growth [2]. Serum samples were collected for NGAL just before antibiotic treatment. It can be useful to check the values of NGAL on the third day and on the $10^{\text {th }}$ day of infection and compare them with the HGB levels on the same days. The relatively small size of the cohort of children may have affected the results of statistical analysis.

\section{Conclusions}

In this study, anemia of inflammation was found in half of the children with UTI, including in those with non-febrile UTI. Delayed onset of anemia was observed in one fifth of children with IA. NGAL was not useful for prediction of IA in children with UTI since its diagnostic profile for predicting anemia was inferior to conventional inflammatory markers. Higher levels of inflammatory markers and longer duration of fever in children with anemia confirmed the relationship between the severity of UTI and the occurrence and severity of IA.

The authors declare no conflict of interest.

\section{References}

1. Fraenkel PG (2017): Anemia of inflammation: a review. Med Clin North Am 101: 285-296.

2. Ganz T, Geffen D (2009): Iron sequestration and anemia of inflammation. Semin Hematol 46: 387-393.

3. Lee JH, Yim HE, Yoo KH (2020): Associations of plasma neutrophil gelatinase-associated lipocalin, anemia, and renal scarring in children with febrile urinary tract infections. J Korean Med Sci 35: e65.

4. Sipahi T, Köksal T, Tavil B, et al. (2004): The effects of acute infection on hematological parameters. Pediatr Hematol Oncol 21: 511-518.

5. Choi JW, Fujii T, Fujii N (2016): Elevated plasma neutrophil gelatinase-associated lipocalin level as a risk factor for anemia in patients with systemic inflammation. Biomed Res Int 2016: 9195219

6. Jayaweera JAAS, Reyes M, Jiseph A (2019): Chidhood iron deficiency anemia leads to recurrent respiratory tract infections and gastroenteritis. Sci Rep 9: 12637.

7. Papanikolaou G, Pantopoulos K (2017): Systemic iron homeostasis and erythropoiesis. IUBMB Life 69: 399-413.

8. Cui LY, Yang S, Zhang J (2011): Protective effect of neutrophil gelatinase-associated lipocalin on hypoxia/reoxygenation injury of HK-2 Cells. Transplant Proc 43: 3622-3627.

9. Nasioudis D, Witkin SS (2015): Neutrophil gelatinase-associated lipocalin and innate immune responses to bacterial infections. Med Microbiol Immunol 204: 471-479.

10. Clerico A, Galli C, Fortunato A, et al. (2012): Neutrophil gelatinase associated lipocalin (NGAL) as biomarker of acute kidney injury: a review of the laboratory characteristics and clinical evidences. Clin Chem Lab Med 50: 1505-1517.

11. Miharada K, Hiroyama T, Sudo K, et al. (2005): Lipocalin 2 functions as a negative regulator of red blood cell production in an autocrine fashion. FASEB J 19: 1881-1883. 
12. Bolignano D, Coppolino G, Donato V, et al. (2010): Neutrophil gelatinase-associated lipocalin (NGAL): a new piece of the anemia puzzle? Med Sci Monit 16: RA131-135.

13. Singer E, Markó L, Paragas N, et al. (2013): Neutrophil gelatinase-associated lipocalin: pathophysiology and clinical applications. Acta Physiol (Oxf) 207: 663-672.

14. Filho LT, Grande AJ, Colonetti T, et al. (2017): Accuracy of neutrophil gelatinase-associated lipocalin for acute kidney injury diagnosis in children: systemic review and meta-analysis. Pediatr Nephrol 32: 1979-1988.

15. Bakal Ü, Sarac M, Tartar T, et al. (2019): A study of the utility of novel non-invasive urinary and serum biomarkers of blunt kidney injury in a rat model: NGAL, KIM-1, and IL-18. Centr Eur J Immunol 44: 219-225.

16. Moriya H, Mochida Y, Ishioka K, et al. (2017): Plasma neutrophil gelatinase-associated lipocalin (NGAL) is an indivator of interstitial damage and a predictor of kidney function worsening of chronic kidney disease in the early stage: a pilot study. Clin Exp Nephrol 21: 1053-1059.

17. Jung N, Byun HJ, Park JH, et al. (2018): Diagnostic accuracy of urinary biomarkers in infants younger than 3 months with urinary tract infection. Korean J Pediatr 61: 24-29.

18. Yun BA, Yang EM, Kim CJ (2018): Plasma neutrophil gelatinase-associated lipocalin as a predictor of renal parenchymal involvement in infants with febrile urinary tract infection: a preliminary study. Ann Lab Med 38: 425-430.

19. Krzemień G, Pańczyk-Tomaszewska M, Kotuła I, et al. (2019): Serum neutrophil gelatinase-associated lipocalin for predicting acute pyelonephritis in infants with urinary tract infection. Centr Eur J Immunol 44: 45-50.

20. Leung AKC, Wong AHC, Leung AAM, et al. (2019): Urinary tract infection in children. Recent Pat Inflamm Allergy Drug Discov 13: 2-18.

21. Kidney Disease: Improving Global Outcomes (KDIGO) CKD Work Group (2013): KDIGO 2012 clinical practice guideline for the evaluation and management of chronic kidney disease. Kidney Int Suppl 3: 259-305.

22. Subcommittee on Urinary Tract Infection (2016): Reaffirmation of AAP clinical practice guideline: the diagnosis and management of the initial urinary tract infection in febrile infants and young children 2-24 months of ages. Pediatrics 138: e20163026.

23. Fernbach SK, Maizels M, Conway JJ (1993): Ultrasound grading of hydronephrosis: introduction to the system used by the Society for Fetal Urology. Pediatr Radiol 23: 478-480.

24. Lebowitz RL, Olbing H, Parkkulainen KV, et al. (1985): International reflux study in children: international system of radiographic grading of vesicoureteral reflux. Pediatr Radiol 15: 105-109.

25. Petrovic S, Bogavac-Stanojevic N, Peco-Antic A, et al. (2013): Clinical application neutrophil gelatinase-associated lipocalin and kidney injury molecule- 1 as indicators of inflammation persistence and acute kidney injury in children with urinary tract infection. Biomed Res Int 2013: 947157.

26. Kim BK, Yim HE, Yoo KH (2017): Plasma neutrophil gelatinase-associated lipocalin: a marker of acute pyelonephritis in children. Pediatr Nephrol 32: 477-484.

27. Güneş A, Ece A, Aktar F, et al. (2015): Urinary kidney molecules in children with iron-deficiency anemia. Med Sci Monit 24: 4023-4029. 